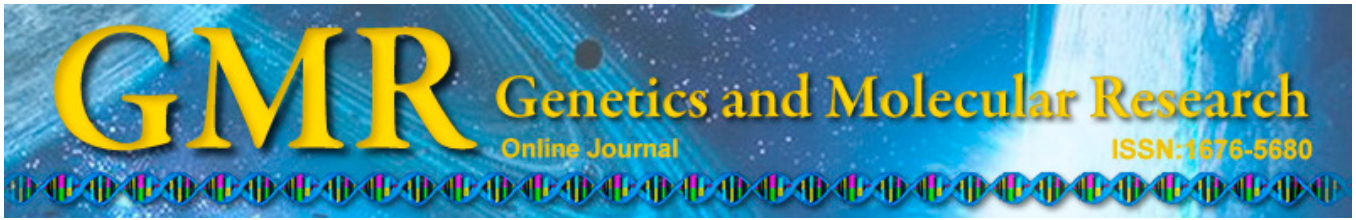

\title{
Translation of foot-and-mouth disease virus RNA: factors influencing alternative AUG selection
}

\author{
J.L. Liu ${ }^{1,2}$, Y.H. Ma ${ }^{3}$, J.Y. Xu' ${ }^{1,2}$, Y.X. Chen ${ }^{1,2}$, S.E. Chen ${ }^{1,2}$ and Z.R. Ma ${ }^{1,2}$ \\ ${ }^{1}$ Key Laboratory of Bioengineering \& Biotechnology of the State Ethnic Affairs \\ Commission, Lanzhou, China \\ ${ }^{2}$ College of Life Science and Engineering, Northwest University for Nationalities, \\ Engineering \& Technology Research Center for Animal Cells, Gansu, China \\ ${ }^{3}$ Gansu Province Center for Disease Prevention and Control, Lanzhou, \\ Gansu, China \\ Corresponding author: Z.R. Ma \\ E-mail: mzr@xbmu.edu.cn
}

Genet. Mol. Res. 14 (4): 16803-16812 (2015)

Received August 15, 2015

Accepted October 26, 2015

Published December 14, 2015

DOI http://dx.doi.org/10.4238/2015.December.14.7

\begin{abstract}
The mechanism of alternative AUG usage in footand-mouth disease virus is not completely understood. Using simple computational approaches, we evaluated the contributions of overall codon bias, quantitative codon bias, and \%GC of the region between the two alternative AUGs, Region-La, as well as the nucleotide bias of the sequence context flanking each AUG with respect to translation initiation efficiency. For all serotypes of this virus, we found that only a small component of the effect of RNA secondary structure on ribosome scanning was due to the low $\% \mathrm{GC}$ of RegionLa. In addition, we found that the nucleotide bias of the context from position -4 to +6 flanking the $\mathrm{AUG}^{2 \text { nd }}$ had a negative correlation with the overall codon bias, and that a strong purine bias existed in this $\mathrm{AUG}^{2 \text { nd }}$ context. However, the quantitative codon bias of RegionLa was seen to be significantly lower than that of Region-Lb (the
\end{abstract}


sequence following $A U G^{2 n d}$ ) in all serotypes except SAT 1-3. Taken together, our results suggest that the low codon bias of Region-La might impair the translation initiation efficiency at the $\mathrm{AUG}^{\mathrm{st}}$ in all serotypes except SAT 1-3, and the specific $\mathrm{AUG}^{2 \text { nd }}$ context might be used as a strong signal to initiate translation from the $\mathrm{AUG}^{2 \text { nd }}$ in all serotypes.

Key words: Codon bias; Foot-and-mouth disease virus; Nucleotide bias; Translation initiation efficiency

\section{INTRODUCTION}

Foot-and-mouth disease virus (FMDV) is a member of the Picornaviridae family. The virus exists as seven serotypes: O, A, C, SAT 1-3, and Asia 1. The virus has a sense RNA genome that functions as an mRNA and encodes a polyprotein (Knowles and Samuel, 2003). The $L$ gene encodes the first component of the polyprotein, is located at the 5 ' terminus of the whole coding sequence, and possesses two potential in-frame initiation codons which generate the Lab and $\mathrm{Lb}$ isoforms (Clarke et al., 1985). The two isoforms possess indistinguishable activities as they both contain the active site residues in the Lb isoform and are involved in the shut-off of host protein synthesis (Devaney et al., 1988). Eukaryotic ribosomes scan mRNA from the $5^{\prime}$ to $3^{\prime}$ ends and usually initiate translation from the first recognized start codon (Kozak, 1983, 1986); however, some reports indicate that ribosomes can "pass over" the first start codon and utilize the second to trigger translation initiation (Kozak, 1989, 1991). High translation initiation efficiency from the 11th AUG in the encephalomyocarditis virus has also been reported (Kaminski et al., 1994). Despite the potential for ribosomes to translate viral proteins from the first start codon, most tend to choose the second AUG as the authentic initiation codon for FMDV, but the reason for this is not completely understood (Cao et al., 1995). Modification of the region between the two FMDV AUGs was not found to enhance the efficiency of translation initiation from the first AUG, and the modified region had no apparent effect on the efficiency of translation initiation from the second (Lopez de Quinto and Martinez-Salas, 1999). These results together suggest that the region flanked by the two AUGs appears to be surplus for FMDV; on the other hand, Piccone et al. (1995) found that this region plays an important role in achieving the translation of viral proteins (Piccone et al., 1995). By analyzing the codon usage pattern of the region between the two AUGs, Zhou et al. $(2010,2013)$ found that the existence of non-preferred codons in this region might have a negative effect on the selection of the first $\mathrm{AUG}\left(\mathrm{AUG}^{\mathrm{st}}\right)$ and contribute to translation initiation at the second $\mathrm{AUG}$ $\left(\mathrm{AUG}^{\text {2nd }}\right.$ ) (Zhou et al., 2010, 2013). However, a systematic analysis of factors potentially important in influencing the efficiency of translation initiation at the two AUGs, such as quantitative codon bias, nucleotide bias, and \%GC, has not yet been applied to FMDV. Here, we employed such analytic methods targeting 96 FMDV isolates and found that the low codon bias of the region between the two AUGs, Region-La, might impair the translation initiation from $A U G^{1 s t}$ in all serotypes except $S A T$ 1-3, and that the specific $\mathrm{AUG}^{2 \text { nd }}$ context (from position -18 through +6 ) can be used as a strong signal to initiate translation from $\mathrm{AUG}^{2 \mathrm{nd}}$ in all serotypes. 


\section{MATERIAL AND METHODS}

\section{Sequence data}

The complete RNA sequences of 96 FMDV isolates were downloaded from the National Center for Biotechnology Information (NCBI) database, (http://www.ncbi.nlm.nih.gov/ Genbank/), including 17 Asia 1, 27 A, 11 C, 26 O, 5 SAT 1, 6 SAT 2, and 4 SAT 3 serotype genomes ( $\underline{\text { Table S1 }})$.

\section{Codon bias detection}

To investigate the codon bias of the coding sequences led by the different AUGs, the formula of the codon adaptation index (CAI) was applied to measure the synonymous codon usage bias (Sharp and $\mathrm{Li}, 1987$ ). CAI was originally proposed to provide a normalized estimate for codon bias, which can be used across the whole coding sequence. The CAI range is from 0 to 1; namely, a higher value reflects a stronger synonymous codon bias. In this study, the CAI data for the whole coding sequences led by the two AUGs were termed $\mathrm{CAI}_{\text {Lab-AUG }}$ and $\mathrm{CAI}_{\mathrm{Lb}-\mathrm{AUG}}$, respectively.

\section{Determination of the GC content and the extent of codon bias in the target regions}

Two regions were targeted for calculation of the GC content (\%GC) and the extent of codon bias (ECB): Region-La and Region-Lb, the latter of which consisted of the sequence downstream of the second AUG. To observe the effects of the \%GC on RNA secondary structure, the \%GCs of Regions-La and -Lb were calculated.

A simple ECB formula was developed to analyze the quantitative codon bias of Regions- $\mathrm{La}$ and $-\mathrm{Lb}$ as well. Because the relative synonymous codon usage (RSCU) is a basic method used to estimate the ratio of the observed to the expected codon frequency given that all synonymous codons for the same amino acids are used equally and that the index is never affected by amino acid usage or the abundance ratio of synonymous codon usage, the RSCU value was used to calculate the $\mathrm{CUB}_{j}$ value using the formula below, in accordance with a previous report (Sharp and $\mathrm{Li}, 1986$ ).

$$
C U B j=|R S C U j-1|
$$

where $\mathrm{CUB}_{i j}$ is an absolute value for the $\mathrm{j}^{\text {th }}$ codons, which belonged to the 59 standard codons excluding ATG, TGG, and the three stop codons at the $i^{\text {th }}$ position of Regions-La or $-\mathrm{Lb}$. The value $i-1$ reflects that the start codon (AUG) at the first position in the two target regions was removed. ECB can be used as a simple and effective measure for the overall codon bias of an open reading frame in the whole coding sequence. It needs to be noted that the values of $\mathrm{CUB}_{\mathrm{AUG}}$ and $\mathrm{CUB}_{\mathrm{UGG}}$ were assigned to zero $\left(\mathrm{CUB}_{\mathrm{AUG}}=0\right.$ and $\mathrm{CUB}_{\mathrm{UGG}}$ $=0$ ); therefore, when the non-authentic start codon (AUG) and UGG for Trp exist in the two regions, it can lead to a problem in calculating ECB. Thus, for calculations, a value of 0.001 is provisionally given to the CUB values of AUG and UGG at these positions to resolve this issue. 


\section{Nucleotide preference calculation of contexts flanking the different start codons}

The relative adaptiveness of a nucleotide $\left(\mathrm{w}_{i j}\right)$ is defined as the usage frequency of the nucleotide at the $i$-th position, compared to the frequency of the optimal nucleotide at the corresponding position. With these $\mathrm{w}_{i j}$ values, the $\mathrm{AUG}$ context adaptation index $\left(\mathrm{A}_{\mathrm{UG}} \mathrm{CAI}\right)$ of the corresponding start codon contexts (from position -18 through +6 ) was used to identify the respective nucleotide usage biases. The formula of the $\mathrm{A}_{\mathrm{UG}} \mathrm{CAI}$ is similar to that of the CAI for codon bias and is calculated as a geometric mean of the $\mathrm{w}_{i j}$ values (Miyasaka, 1999). The AUG contexts flanking $\mathrm{AUG}^{\text {sst }}$ and $\mathrm{AUG}^{\text {2nd }}$ were, respectively, compiled with manual editing, and the relative adaptiveness of the nucleotide $\left(\mathrm{w}_{i j} ; \mathrm{I}=-18\right.$ through $+6, j=\mathrm{A}, \mathrm{U}, \mathrm{C}$, or $\left.\mathrm{G}\right)$ at each position was used to estimate the nucleotide usage bias of the two contexts.

\section{Comparison and correlation analyses}

To investigate the effect of the codon bias of the two target regions on the translation initiation efficiency of the corresponding coding sequences, the $\mathrm{ECB}_{\text {Region-La }}$ and $\mathrm{ECB}_{\text {Region-Lb }}$ data were compared using one-way ANOVA. For testing the effect of the folding energy caused by the $\% \mathrm{GC}$ of the two regions on alternative $\mathrm{AUG}$ usage, the $\% \mathrm{GC}_{\text {Region-La }}$ and $\% \mathrm{GC}_{\text {Region-Lb }}$ data were also compared by one-way ANOVA. To detect the effect of the context at positions -18 to +6 flanking each of the two start codons on the translation initiation efficiency, the $\mathrm{A}_{\mathrm{UG}} \mathrm{CAI}_{\text {Region-La }}$ and $\mathrm{A}_{\mathrm{UG}} \mathrm{CAI}_{\text {Region-Lb }}$ data were compared by one-way ANOVA.

To examine the relationship between the factors of the nucleotides flanking the AUGs and the overall codon bias of the whole coding sequence, correlation analyses between the $\mathrm{A}_{\mathrm{UG}} \mathrm{CAIs}$ for the serial deletion of positions (from -18 to $+6,-17$ to +6 , etc. through -4 to +6 ) of the two start codon contexts and the corresponding CAIs were implemented using Pearson's rank method.

\section{RESULTS}

The $\mathrm{CAI}_{\mathrm{Lab}-\mathrm{AUG}}$ and $\mathrm{CAI}_{\mathrm{Lb}-\mathrm{AUG}}$ measures calculated in this study, which ranged from 0.212 to 0.240 with an average of 0.226 and standard deviation (SD) of 0.006 , indicated that low codon bias existed in the FMDV virus (Table S2). No significant difference in codon bias was identified between the whole coding sequence led by either $\mathrm{AUG}^{1 \text { st }}$ or $\mathrm{AUG}^{2 \mathrm{nd}}(\mathrm{P}=$ 0.77), suggesting that the overall codon bias had no effect on alternative AUG usage. It has been generally accepted that FMDV populations consist of distributions of related but varied genomes due to high mutation rates (Domingo et al., 2003). Such high mutation rates might be the reason why the overall codon bias is weak in FMDV. The characteristic of low codon bias might not aid viruses to efficiently replicate in susceptible host cells, but might help the virus adapt to potentially distinct codon preferences of the host cell.

We found that the \%GC of Region-La was significantly lower than that of Region-Lb $\left(\mathrm{P}=3.22 \times 10^{-23}\right)$ (Table S3), implying that the degree of stability of Region-La RNA secondary structure was weaker than that of Region-Lb, and that the folding structure of Region-La might have little effect on impairing ribosomes scanning along this region, since ribosomes can scan along Region-Lb smoothly. According to an analysis of the translation efficiency of green fluorescent protein (GFP) protein in Escherichia coli, the \%GC near the start codon 
was strongly correlated with the expression level; this effect was likely mediated by mRNA secondary structure caused by the GC level (Kudla et al., 2009). A stable hairpin structure in Region-La might enhance translation initiation efficiency at the second AUG of FMDV (Hinton et al., 2000), whereas the translation initiation efficiency of the second start codon would likely be impaired if ribosomes were blocked in Region-La (Pöyry et al., 2001). It implies that ribosomes need to scan Region-La to trigger translation initiation from $\mathrm{AUG}^{\text {2nd }}$. Since, as determined in this study, the \%GC of Region-La is lower than that of Region-Lb, the mRNA secondary structure caused by the \%GC of Region-La is likely less stable than is that of Region-Lb. We suggest that the RNA secondary structure of Region-La therefore never serves to block ribosomal scanning across this region, and that this is another potential factor for regulating translation initiation by preferential ribosomal recognition of the second start codon.

The CUB values of 59 standard codons in the FMDV open reading frame (ORF) are listed in Table S4 and were used to calculate the ECB data (Table S3), which were in turn used to evaluate the quantitative codon biases of Regions-La and - $\mathrm{Lb}$ of the seven FMDV serotypes. For each of the Asia 1, A, C, and O serotypes, the codon bias of Region-La was significantly lower than that of Region-Lb (Figure 1). For each of the serotypes SAT 1-3, there were no significant differences in codon bias between Regions (Figure 1).

These results indicated that the influence of codon bias in Region-La on the use of alternative start codons in serotypes SAT 1-3 is different than that in serotypes Asia 1, A, C, and $\mathrm{O}$. This difference might be due to the fact that serotypes Asia 1, A, C, and $\mathrm{O}$ are clearly distinct from serotypes SAT 1-3 in their evolutionary characteristics (Lewis-Rogers et al., 2008). Researchers introduced two additional AUGs into FMDV Region-La, and found that the ribosomes never registered the additional AUGs and still had a tendency to initiate translation from the original second AUG (Belsham, 1992). This indicated that Region-La cannot provide an optimal environment for ribosomes scanning, due to its low codon bias. According to the view of "selection and mutation-drift" for codon bias (Sharp and Li, 1986), codon bias can reflect the balance between selection favoring preferred codons and mutation-drift causing persistence of non-preferred codons: translation selection is a main factor in highly expressed genes, whereas mutation-drift dominates codon bias in poorly expressed genes. A previous report on the comparative genomics of FMDV indicated that the mutation rate of Region-La was higher than that of Region-Lb (Carrillo et al., 2005). To date, the different efficiencies of translation initiation at the two start codons has only been studied for the FMDV serotypes O and A. The lack of difference in codon biases between Regions-La and -Lb in serotypes SAT 1-3 might be due to the apparent distinction of their evolutionary characteristics from those of the other four serotypes. The low codon bias of Region-La in the Asia 1, A, C, and O serotypes might be a factor for impairing initiation translation from the first AUG in these serotypes, and for assisting ribosomes in recognizing $\mathrm{AUG}^{2 \text { nd }}$ to initiate translation.

Some reports have shown that the specific nucleotide context flanking the authentic start codon of a gene can act as a strong signal of translation initiation to achieve protein synthesis, and that the efficiency of translation initiation at the original start codon decreases when the particular context is modified (Deana et al., 1998; Sato et al., 2001). To evaluate the correlation between the nucleotide bias of the context flanking each AUG and the expression level of the FMDV RNA, both $\mathrm{A}_{\mathrm{UG}} \mathrm{CAI}_{\mathrm{AUG}}{ }^{\text {st }}$ and $\mathrm{A}_{\mathrm{UG}} \mathrm{CAI}_{\mathrm{AUG}}{ }^{2 \text { nd }}$ values were calculated and plotted against $\mathrm{CAI}_{\mathrm{Lab}-\mathrm{AUG}}$ and $\mathrm{CAI}_{\mathrm{Lb}-\mathrm{AUG}}$ values, respectively. We identified a positive correlation between $\mathrm{CAI}_{\text {Lab-AUG }}$ and the specific $\mathrm{AUG}^{\text {st }}$ context (from position -16 to +6 ) [simple linear correlation 
coefficient $r=0.34, P=0.001\left(r^{2}=0.12\right)$ ] (Figure 2). However, a negative correlation was observed between $\mathrm{CAI}_{\mathrm{Lb}-\mathrm{AUG}}$ and the specific $\mathrm{AUG}^{2 n d}$ context (from position -4 to +6 ) [simple linear correlation coefficient $r=-0.21, P=0.043\left(r^{2}=0.09\right)$ ] (Figure 3$)$. As the specific $\mathrm{AUG}^{\text {2nd }}$ context had a negative correlation with the overall codon bias and the latter was low, the specific $\mathrm{AUG}^{2 \text { nd }}$ context might be predicted to enhance the translation initiation efficiency at $\mathrm{AUG}^{2 \mathrm{nd}}$.

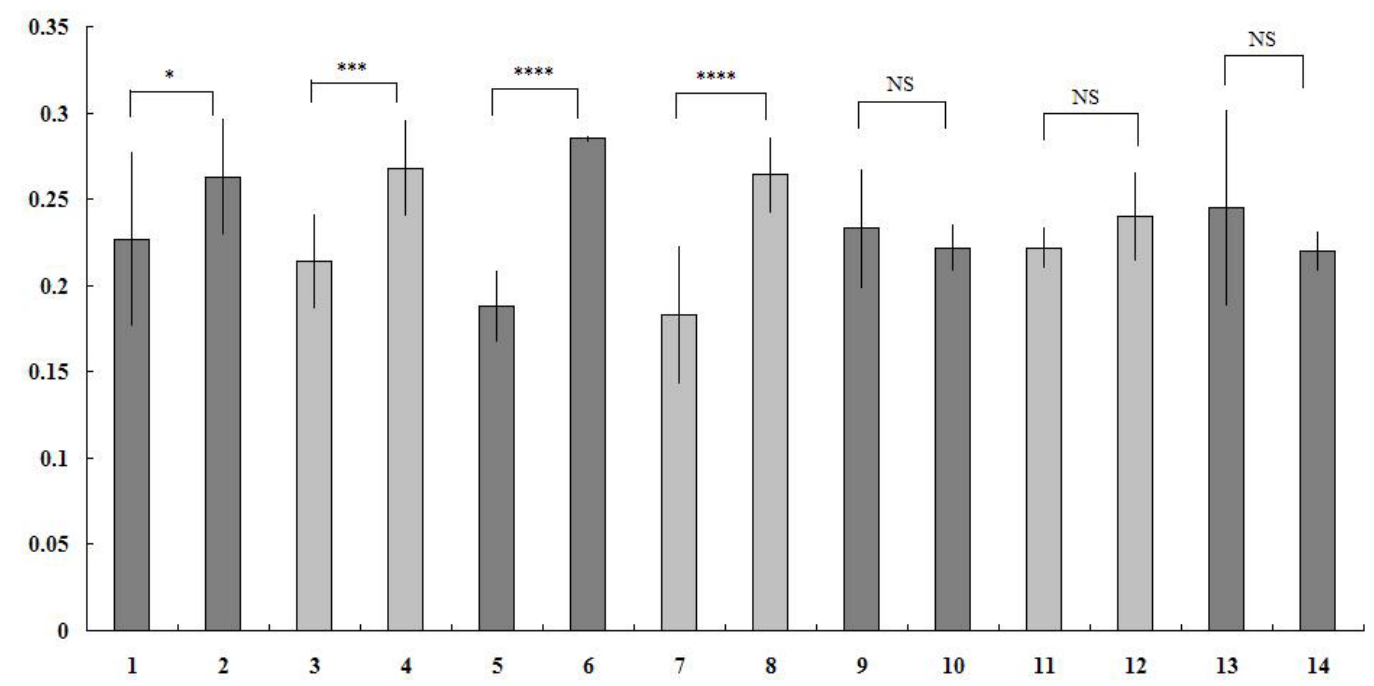

Figure 1. The difference of quantitative codon bias between the Region-La and the Region-Lb of FMDV. No. 1, 3, 5, 7, 9, 11, 13 show $\mathrm{ECB}_{\text {the Region-La }}$ data for serotypes Asia 1, A, C, O, SAT 1, SAT 2, SAT 3 of FMDV. No. 2, 4, 6, 8, $10,12,14$ show $\mathrm{ECB}_{\text {the Region-Lb }}$ data for corresponding serotypes above. The asterisk $\left(^{*}\right)$ means $\mathrm{P}<0.05, * * *$ means $\mathrm{P}<10^{-8}, * * * *$ means $\mathrm{P}<10^{-11}$. NS show $\mathrm{P}>0.05$.

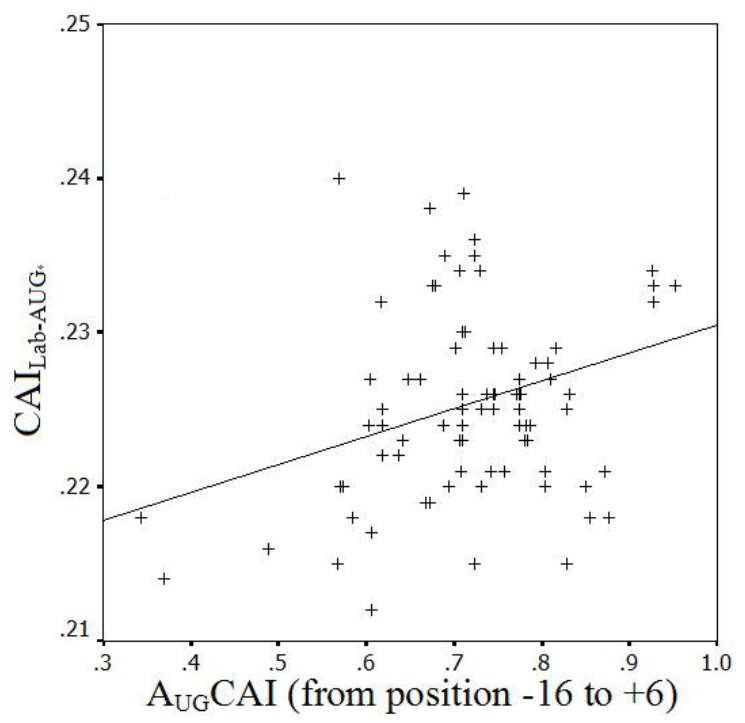

Figure 2. Correlation between the nucleotide bias of the $\mathrm{AUG}^{1 \mathrm{st}}$ specific context and the overall codon bias of coding sequence led by the $\mathrm{AUG}^{\text {1st }}$ of FMDV. 


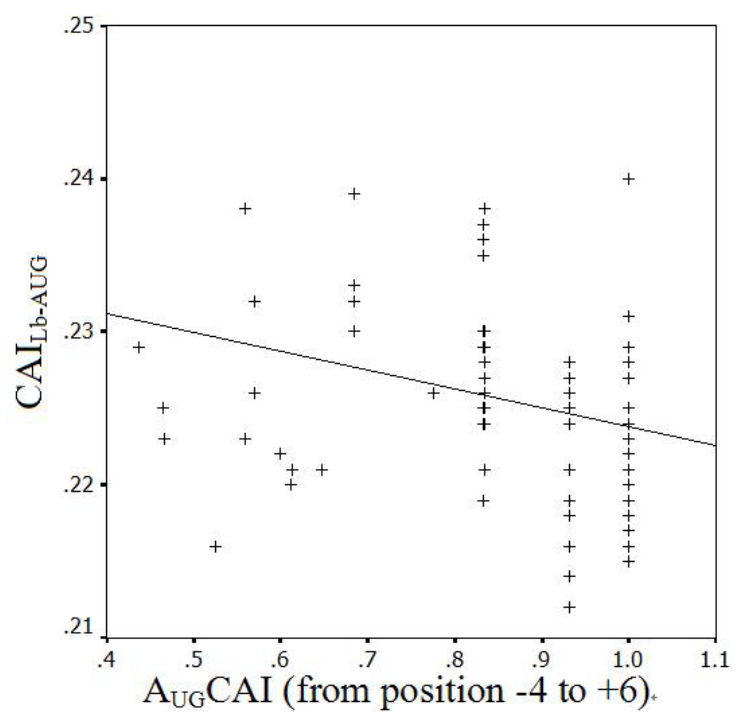

Figure 3. Correlation between the nucleotide bias of the $\mathrm{AUG}^{2 \text { nd }}$ specific context and the overall codon bias of coding sequence led by the $\mathrm{AUG}^{2 \mathrm{nd}}$ of FMDV.

By analyzing the six positions (from -3 to -1 and from +4 to +6 ) flanking each AUG, we determined that a strong bias existed for particular nucleotides flanking both AUGs. For the $\mathrm{AUG}^{1 \mathrm{st}}$ context, the nucleotide $\mathrm{U}$ was preferentially chosen in positions $-3,-2$, and -1 , and the nucleotide $A$ was preferentially used in positions +4 and +5 (Table 1 ). For the $\mathrm{AUG}^{2 \mathrm{nd}}$ context, the nucleotides A or $\mathrm{G}$ were preferentially chosen in positions -3 and -2 , and nucleotide $\mathrm{G}$ in position +4 and nucleotide $\mathrm{A}$ in the positions +5 and +6 showed the strongest bias (Table 2). In addition, the nucleotides in position -1 flanking $\mathrm{AUG}^{2 \mathrm{nd}}$ were exclusively purines (G: 95/96 and A: 1/96) (Table 2). These results suggested that the positions flanking $\mathrm{AUG}^{2 \mathrm{nd}}$ play important roles in its recognition by eukaryotic ribosomes as an authentic start codon. Translation initiation at the start codon in the whole coding sequence of FMDV requires recognition of the context of the AUG as an important signal to trigger translation initiation. The sequence contexts flanking start codons have a significant correlation with the efficiency of translation initiation across various organisms (Hamilton et al., 1987; Cavener and Ray, 1991; Ikeda and Miyasaka, 1998; Miyasaka, 1999, 2002), in agreement with our findings. Compared with the strong bias of usage of nucleotide $U$ in positions -2 and -3 flanking the $\mathrm{AUG}^{\text {sst }}$, we found that the corresponding positions flanking the $\mathrm{AUG}^{2 \mathrm{nd}}$ preferentially incorporated purine nucleotides. Furthermore, a strong bias for incorporation of the A nucleotide existed in both positions +4 and +5 in the $\mathrm{AUG}^{\text {st }}$ context (Table 1), whereas a strong bias for incorporation of $\mathrm{G}$ existed in position +4 and a strong bias for incorporation of A existed in position +5 of $\mathrm{AUG}^{2 \mathrm{nd}}$ (Table 2). This finding implies that under translation selection, purine nucleotides in positions $-2,-3$, and +4 flanking $\mathrm{AUG}^{2 \mathrm{nd}}$, when it is acting as a translation initiation signal, can assist eukaryotic ribosomes in its recognition. Similar nucleotide biases at positions $-2,-3$, and +4 , which result in high translation initiation efficiency at the start codon in various organisms, have also been reported (Kozak, 1986, 1997, 1999; Kochetov, 2005; Pisarev et al., 2006; Nakagawa et al., 
2008). This is the first report that such nucleotide bias at these important positions flanking the AUG also exists in viruses. With respect to the specific AUG ${ }^{\text {nd }}$ context in FMDV, this characteristic can be explained by the supposition that the viral genome forms similar AUG contexts as does the host cell, to adapt to the translation initiation model, under translation selection. Our study indicates that purines in positions -2 and -3 and a $G$ in position +4 in the $\mathrm{AUG}^{2 \mathrm{nd}}$ context can serve as an important signal which assists eukaryotic ribosomes in recognizing the second initiation codon of FMDV.

Table 1. Frequencies and relative adaptiveness (w) of the nucleotide surrounding the first start codon.

\begin{tabular}{|c|c|c|c|c|c|c|c|c|}
\hline Position & A & $\mathrm{U}$ & $\mathrm{C}$ & G & $\mathrm{W}_{\mathrm{A}}$ & $\mathrm{W}_{\mathrm{U}}$ & $\mathrm{W}_{\mathrm{C}}$ & $\mathrm{W}_{\mathrm{G}}$ \\
\hline-18 & 0 & 84 & 12 & 0 & 0 & 1.000 & 0.143 & 0 \\
\hline-17 & 5 & 86 & 5 & 0 & 0.058 & 1.000 & 0.058 & 0 \\
\hline-16 & 26 & 65 & 1 & 4 & 0.400 & 1.000 & 0.015 & 0.062 \\
\hline-15 & 73 & 7 & 16 & 0 & 1.000 & 0.096 & 0.219 & 0 \\
\hline-14 & 28 & 25 & 42 & 1 & 0.667 & 0.595 & 1.000 & 0.024 \\
\hline-13 & 46 & 25 & 24 & 1 & 1.000 & 0.543 & 0.522 & 0.022 \\
\hline-12 & 53 & 14 & 28 & 1 & 1.000 & 0.264 & 0.528 & 0.019 \\
\hline-11 & 34 & 24 & 38 & 0 & 0.895 & 0.632 & 1.000 & 0 \\
\hline-10 & 34 & 28 & 32 & 2 & 1.000 & 0.824 & 0.941 & 0.059 \\
\hline-9 & 61 & 6 & 29 & 0 & 1.000 & 0.098 & 0.475 & 0 \\
\hline-8 & 15 & 30 & 51 & 0 & 0.294 & 0.588 & 1.000 & 0 \\
\hline-7 & 17 & 65 & 12 & 2 & 0.262 & 1.000 & 0.185 & 0.031 \\
\hline-6 & 18 & 15 & 19 & 44 & 0.409 & 0.341 & 0.432 & 1.000 \\
\hline-5 & 59 & 31 & 3 & 3 & 1.000 & 0.525 & 0.051 & 0.051 \\
\hline-4 & 18 & 44 & 34 & 0 & 0.410 & 1.000 & 0.773 & 0 \\
\hline-3 & 8 & 75 & 13 & 0 & 0.107 & 1.000 & 0.173 & 0 \\
\hline-2 & 11 & 62 & 21 & 2 & 0.177 & 1.000 & 0.339 & 0.032 \\
\hline-1 & 27 & 46 & 23 & 0 & 0.587 & 1.000 & 0.500 & 0 \\
\hline AUG & - & - & - & - & - & - & - & - \\
\hline+4 & 88 & 0 & 0 & 8 & 1.000 & 0 & 0 & 0.091 \\
\hline+5 & 79 & 0 & 0 & 17 & 1.000 & 0 & 0 & 0.215 \\
\hline+6 & 1 & 31 & 52 & 12 & 0.019 & 0.596 & 1.000 & 0.231 \\
\hline
\end{tabular}

\section{Table 2. Frequencies and relative adaptiveness (w) of the nucleotide surrounding the second start codon.}

\begin{tabular}{|c|c|c|c|c|c|c|c|c|}
\hline Position & A & $\mathrm{U}$ & $\mathrm{C}$ & G & $\mathrm{W}_{\mathrm{A}}$ & $\mathrm{W}_{\mathrm{u}}$ & $\mathrm{W}_{\mathrm{C}}$ & $\mathrm{W}_{\mathrm{G}}$ \\
\hline-18 & 10 & 61 & 21 & 4 & 0.164 & 1.000 & 0.344 & 0.066 \\
\hline-17 & 1 & 18 & 77 & 0 & 0.013 & 0.234 & 1.000 & 0 \\
\hline-16 & 75 & 3 & 12 & 6 & 1.000 & 0.040 & 0.160 & 0.080 \\
\hline-15 & 17 & 0 & 77 & 0 & 0.247 & 0 & 1.000 & 0 \\
\hline-14 & 21 & 0 & 0 & 75 & 0.280 & 0 & 0 & 1.000 \\
\hline-13 & 50 & 1 & 3 & 42 & 1.000 & 0.020 & 0.060 & 0.840 \\
\hline-12 & 52 & 1 & 14 & 29 & 1.000 & 0.019 & 0.269 & 0.558 \\
\hline-11 & 13 & 5 & 73 & 5 & 0.178 & 0.068 & 1.000 & 0.068 \\
\hline-10 & 50 & 15 & 24 & 7 & 1.000 & 0.300 & 0.480 & 0.140 \\
\hline-9 & 38 & 0 & 43 & 15 & 0.884 & 0 & 1.000 & 0.349 \\
\hline-8 & 57 & 3 & 33 & 3 & 1.000 & 0.053 & 0.579 & 0.053 \\
\hline-7 & 77 & 3 & 11 & 5 & 1.000 & 0.039 & 0.143 & 0.065 \\
\hline-6 & 16 & 0 & 6 & 74 & 0.216 & 0 & 0.081 & 1.000 \\
\hline-5 & 7 & 0 & 0 & 89 & 0.079 & 0 & 0 & 1.000 \\
\hline-4 & 70 & 2 & 2 & 22 & 1.000 & 0.029 & 0.029 & 0.314 \\
\hline-3 & 93 & 0 & 0 & 3 & 1.000 & 0 & 0 & 0.032 \\
\hline-2 & 83 & 0 & 3 & 10 & 1.000 & 0 & 0.036 & 0.120 \\
\hline-1 & 39 & 0 & 0 & 57 & 0.684 & 0 & 0 & 1.000 \\
\hline AUG & - & - & - & - & - & - & - & - \\
\hline+4 & 1 & 0 & 0 & 95 & 0.011 & 0 & 0 & 1.000 \\
\hline+5 & 95 & 0 & 0 & 1 & 1.000 & 0 & 0 & 0.011 \\
\hline+6 & 75 & 0 & 0 & 21 & 1.000 & 0 & 0 & 0.280 \\
\hline
\end{tabular}




\section{DISCUSSION}

Our results suggest that the characteristics of the specific context flanking the $\mathrm{AUG}^{2 \mathrm{nd}}$ of FMDV lead to the high efficiency of translation initiation at $\mathrm{AUG}^{\text {2nd }}$. Although FMDV possesses a high mutation rate, the specific nucleotide usage pattern flanking $\mathrm{AUG}^{\text {2nd }}$ is generated under translation selection to adapt to the eukaryotic translation mechanism of host cells.

\section{Conflicts of interest}

The authors declare no conflict of interest.

\section{ACKNOWLEDGMENTS}

Research supported by the Program for Changjiang Scholars and Innovative Research Team in University (\#IRT13091), and by the Central Universities deriving from the Northwest University for Nationalities (\#ZYZ2012067).

\section{Supplementary material}

\section{REFERENCES}

Belsham GJ (1992). Dual initiation sites of protein synthesis on foot-and-mouth disease virus RNA are selected following internal entry and scanning of ribosomes in vivo. EMBO J. 11: 1105-1110.

Cao X, Bergmann IE, Füllkrug R and Beck E (1995). Functional analysis of the two alternative translation initiation sites of foot-and-mouth disease virus. J. Virol. 69: 560-563.

Carrillo C, Tulman ER, Delhon G, Lu Z, et al. (2005). Comparative genomics of foot-and-mouth disease virus. J. Virol. 79: 6487-6504.

Cavener DR and Ray SC (1991). Eukaryotic start and stop translation sites. Nucleic Acids Res. 19: 3185-3192.

Clarke BE, Sangar DV, Burroughs JN, Newton SE, et al. (1985). Two initiation sites for foot-and-mouth disease virus polyprotein in vivo. J. Gen. Virol. 66: 2615-2626.

Deana A, Ehrlich R and Reiss C (1998). Silent mutations in the Escherichia coli ompA leader peptide region strongly affect transcription and translation in vivo. Nucleic Acids Res. 26: 4778-4782.

Devaney MA, Vakharia VN, Lloyd RE, Ehrenfeld E, et al. (1988). Leader protein of foot-and-mouth disease virus is required for cleavage of the p220 component of the cap-binding protein complex. J. Virol. 62; 4407-4409.

Domingo E, Escarmis C, Baranowski E, Ruiz-Jarabo CM, et al. (2003). Evolution of foot-and-mouth disease virus. Virus Res. 91: 47-63.

Hamilton R, Watanabe CK and de Boer HA (1987). Compilation and comparison of the sequence context around the AUG startcodons in Saccharomyces cerevisiae mRNAs. Nucleic Acids Res. 15: 3581-3593.

Hinton TM, Li F and Crabb BS (2000). Internal ribosomal entry site-mediated translation initiation in equine rhinitis A virus: similarities to and differences from that of foot-and-mouth disease virus. J. Virol. 74: 11708-11716.

Ikeda K and Miyasaka H (1998). Compilation of mRNA sequences surrounding the AUG translation initiation codon in the green alga Chlamydomonas reinhardtii. Biosci. Biotechnol. Biochem. 62: 2457-2459.

Kaminski A, Belsham GJ and Jackson RJ (1994). Translation of encephalomyocarditis virus RNA: parameters influencing the selection of the internal initiation site. EMBOJ. 13;1673-1681.

Knowles NJ and Samuel AR (2003). Molecular epidemiology of foot-and-mouth disease virus. Virus Res. 91: 65-80.

Kochetov AV (2005). AUG codons at the beginning of protein coding sequences are frequent in eukaryotic mRNAs with a suboptimal start codon context. Bioinformatics 21: 837-840.

Kozak M (1983). Comparison of initiation of protein synthesis in procaryotes, eucaryotes, and organelles. Microbiol. Rev. 47: $1-45$.

Kozak M (1986). Point mutations define a sequence flanking the AUG initiator codon that modulates translation by eukaryotic ribosomes. Cell 44: 283-292. 
Kozak M (1989). The scanning model for translation: an update. J. Cell Biol. 108: 229-241.

Kozak M (1991). A short leader sequence impairs the fidelity of initiation by eukaryotic ribosomes. Gene Expr. 1: 111-115.

Kozak M (1997). Recognition of AUG and alternative initiator codons is augmented by $\mathrm{G}$ in position +4 but is not generally affected by the nucleotides in positions +5 and +6 . EMBO J. 16: 2482-2492.

Kozak M (1999). Initiation of translation in prokaryotes and eukaryotes. Gene 234: 187-208.

Kudla G, Murray AW, Tollervey D and Plotkin JB (2009). Coding-sequence determinants of gene expression in Escherichia coli. Science 324: 255-258.

Lewis-Rogers N, McClellan DA and Crandall KA (2008). The evolution of foot-and-mouth disease virus: impacts of recombination and selection. Infect. Genet. Evol. 8: 786-798.

López de Quinto S and Martinez-Salas E (1999). Involvement of the aphthovirus RNA region located between the two functional AUGs in start codon selection. Virology 255: 324-336.

Miyasaka H (1999). The positive relationship between codon usage bias and translation initiation AUG context in Saccharomyces cerevisiae. Yeast 15: 633-637.

Miyasaka H (2002). Translation initiation AUG context varies with codon usage bias and gene length in Drosophila melanogaster. J. Mol. Evol. 55: 52-64.

Nakagawa S, Niimura Y, Gojobori T, Tanaka H, et al. (2008). Diversity of preferred nucleotide sequences around the translation initiation codon in eukaryote genomes. Nucleic Acids Res. 36: 861-871.

Piccone ME, Rieder E, Mason PW and Grubman MJ (1995). The foot-and-mouth disease virus leader proteinase gene is not required for viral replication. J. Virol. 69: 5376-5382.

Pisarev AV, Kolupaeva VG, Pisareva VP, Merrick WC, et al. (2006). Specific functional interactions of nucleotides at key -3 and +4 positions flanking the initiation codon with components of the mammalian $48 \mathrm{~S}$ translation initiation complex. Genes Dev. 20: 624-636.

Pöyry TA, Hentze MW and Jackson RJ (2001). Construction of regulatable picornavirus IRESes as a test of current models of the mechanism of internal translation initiation. RNA 7: 647-660.

Sato T, Terabe M, Watanabe H, Gojobori T, et al. (2001). Codon and base biases after the initiation codon of the open reading frames in the Escherichia coli genome and their influence on the translation efficiency. J. Biochem. 129: 851-860.

Sharp PM and Li WH (1986). An evolutionary perspective on synonymous codon usage in unicellular organisms. J. Mol. Evol. 24: 28-38.

Sharp PM and Li WH (1987). The codon Adaptation Index - a measure of directional synonymous codon usage bias, and its potential applications. Nucleic Acids Res. 15: 1281-1295.

Zhou JH, Zhang J, Ding YZ, Chen HT, et al. (2010). Characteristics of codon usage bias in two regions downstream of the initiation codons of foot-and-mouth disease virus. Biosystems 101: 20-28.

Zhou JH, You YN, Chen HT, Zhang J, et al. (2013). The effects of the synonymous codon usage and tRNA abundance on protein folding of the 3C protease of foot-and-mouth disease virus. Infect. Genet. Evol. 16: 270-274. 